\title{
A light and electron microscopy study of the yak placentome
}

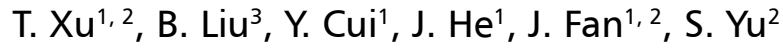 \\ ${ }^{1}$ Academic of Veterinary Medicine, Gansu Agricultural University, Lanzhou, China \\ ${ }^{2} T e c h n o l o g y$ and Research Centre of Gansu Province for Embryonic Engineering of Bovine \\ and Sheep and Goat, Lanzhou, China \\ ${ }^{3}$ College of Life Science and Resources and Environment, Yichun University, Yichun, China
}

[Received: 25 December 2018; Accepted: 7 February 2019]

Background and Materials and methods: In order to clarify and reveal the morphological characteristics of yak placentomes, placentomes obtained from 151 to 180 days of pregnant yaks were observed using light microscopy and scanning and transmission electron microscopy.

Results: The results indicated that sessile, dome-shaped yak placentomes seemed to have a relatively complex villous-crypt architecture pattern. There was a straight maternal plate beneath the placentome. Plentiful uterine glands and a dense cellular layer were present in the endometrium lamina propria close to the maternal plate. Trophoblast giant cells appeared to have similar ultrastructure features to these in other ruminants, including abundant mitochondria, an extensive array of rough endoplasmic reticulum, advanced Golgi complex and many specific secretory granules. Trophoblast giant cells could also secrete neutral and acid glycoconjugates. Furthermore, numerous glycoconjugates were distributed in the connective zones between mononuclear trophoblast cells and crypt epithelial cells as well as in maternal connective tissues. Mononucleate trophoblast cells, which had abundant microvilli that interdigitated with the corresponding microvilli arising from the crypt epithelial cells, had numerous mitochondria and vesicles, but there were no glycoconjugates.

Conclusions: The morphological structures of yak placentomes were similar to those of other bovid genera; however, certain differences were observed. These findings might provide morphological evidence for evolutionary relationships between different bovid genera. (Folia Morphol 2019; 78, 4: 818-826)

Key words: yak, placentome, microstructure, ultrastructure, histochemistry

\section{INTRODUCTION}

The placenta is the link between the mother and foetus during pregnancy. It is not only a place for material and gas exchange between the mother and foetus but also a temporary organ with multiple functions [17]. The normal development of placental morphology and function during pregnancy is closely related to the survival and development of the foetus $[4,18]$, and placental abnormalities are often associated with abortion and perinatal mortality.

Yaks (Bos grunniens) are essential livestock for people living in high altitude areas in Central Asia. They are well adapted to the harsh environment at high altitude, low temperature and hypoxia. How-

Address for correspondence: Dr. S. Yu, Technology and Research Centre of Gansu Province for Embryonic Engineering of Bovine and Sheep and Goat, Lanzhou, China, e-mail: sjyu@163.com 
ever, the reproductive efficiencies of yaks are low. They reach puberty late, at a mean age of $33.4 \pm 6.0$ months, and most yak cows calve only once every 2 years. Yaks are seasonally polyoestrous animals. The breeding season starts from July to October, and the calving season occurs from April to July, with the duration of pregnancy ranging from 248 to 258 days [29]. The mean gestation lengths are obviously shorter when compared with other bovids, such as 343 days for African buffalo (Syncerus caffer) [10], 315 days for water buffalo (Bubalus bubalis) and 280 days for cattle (Bos taurus) [7]. With regard to the reproductive organs, some differences are evident between yak and dairy cow. In yak, for example, there are three transverse circles on the uterine cervix, consisting of many tight folds, and the body of the uterus is rather short [3].

There is, however, no report on the microscopic and ultrastructural structure of the yak placentome. In the present study, the placentomes from 6 pregnant yaks were collected to investigate the microscopic and ultrastructural characteristics. The distribution pattern of the glycoconjugates was determined by histochemical techniques. Meanwhile, the authors try to show whether the morphology of the yak placentome is similar to that of other bovid genera or not.

\section{MATERIALS AND METHODS}

\section{Animals}

Six female yaks from 6 to 7 years of age, with the breeding season ranging from July to August, were included in the investigation. The yaks were purchased from an abattoir in Xining City of Qinghai Province as approved by the Welfare Authority of the local government. The experimental yaks were slaughtered at the abattoir; immediately after collection, placentomes were chosen and fixed. The foetal crown-rump lengths of the 6 yaks were $42.0 \mathrm{~cm}, 42.3 \mathrm{~cm}, 44.0 \mathrm{~cm}$, $44.7 \mathrm{~cm}, 45.6 \mathrm{~cm}$ and $47.8 \mathrm{~cm}$, respectively.

\section{Light microscopy}

Three placentomes of the same area of the pregnant horn (close to the foetus) from each yak were randomly selected and further processed for histological and histochemical analysis. The samples were cut into pieces no larger than $1 \mathrm{~cm}^{3}$, fixed in $4 \%$ neutral paraformaldehyde phosphate buffer (PH 7.4) for $24 \mathrm{~h}$, dehydrated in graded alcohols, and embedded in paraffin wax. Vertical sections $4-\mu \mathrm{m}$ thick were cut and stained using a standard haematoxylin and eosin
(H\&E) protocol, periodic acid Schiff (PAS) reaction, Alcian blue (AB, pH 2.5) and AB-PAS (pH 2.5). Light microscopy observations and section image acquisition were carried out using an Olympus DP71 Light Microscope (including DP control and Image-Pro Express, Japan).

\section{Transmission electron microscopy}

For transmission electron microscopy, tissue blocks of approximately $1 \mathrm{~mm}^{3}$ were taken from the centre of each placentome, then immersed in $2.5 \%$ glutaraldehyde in neutral phosphate buffer $\left(\mathrm{pH} 7.4\right.$, at $\left.4^{\circ} \mathrm{C}\right)$ and post-fixed in $1 \%$ osmium tetroxide for $2 \mathrm{~h}$. Samples were dehydrated in a graded series of ethanol and embedded in Epon812. Semithin sections $(1 \mu \mathrm{m})$ were cut and stained with toluidine blue. Suitable regions of interest were chosen, and ultrathin sections (60$-80 \mathrm{~nm}$ ) were cut on a Leica EM-UC6 ultramicrotome, mounted on G200 grids, stained with uranyl acetate and lead citrate and viewed with a JEOL 1230 electron microscope at $120 \mathrm{kV}$.

\section{Scanning electron microscope}

For scanning electron microscopy (SEM), single villous trees were carefully separated from the chorionic plate and further processed for SEM to obtain the triaxial image of villous trees. The samples were immersed in $2.5 \%$ glutaraldehyde in neutral phosphate buffer $\left(\mathrm{pH} 7.4\right.$, at $\left.4^{\circ} \mathrm{C}\right)$ for a minimum of $24 \mathrm{~h}$ and post-fixed in $1 \%$ osmium tetroxide for $1.5 \mathrm{~h}$. Then, the samples were dehydrated in a graded series of ethanol, dried in a critical point drying apparatus using liquid $\mathrm{CO}_{2}$, coated with gold, and imaged using a JEM-100CX scanning electron microscope.

\section{RESULTS}

Anatomical observation of yak placenta

Visually, the yak placenta was multi-cotyledonary, including multiple independent placentomes and an interplacentome. The placentomes were round, oval or irregular, arranged into three to four columns in the uterine horn, and the placentomes at the centre of the uterus were well developed (Fig. 1A). The placentome was divided into uterine caruncles and cotyledons. Uterine caruncles had a convex dome shape with neatly arranged caruncular crypts. Cotyledons were clustered near the foetal villi, which protruded within the corresponding caruncular crypts (Fig. 1B). From the longitudinal section of the placentome, the foetal tissue formed a convex chorion plate (foe- 

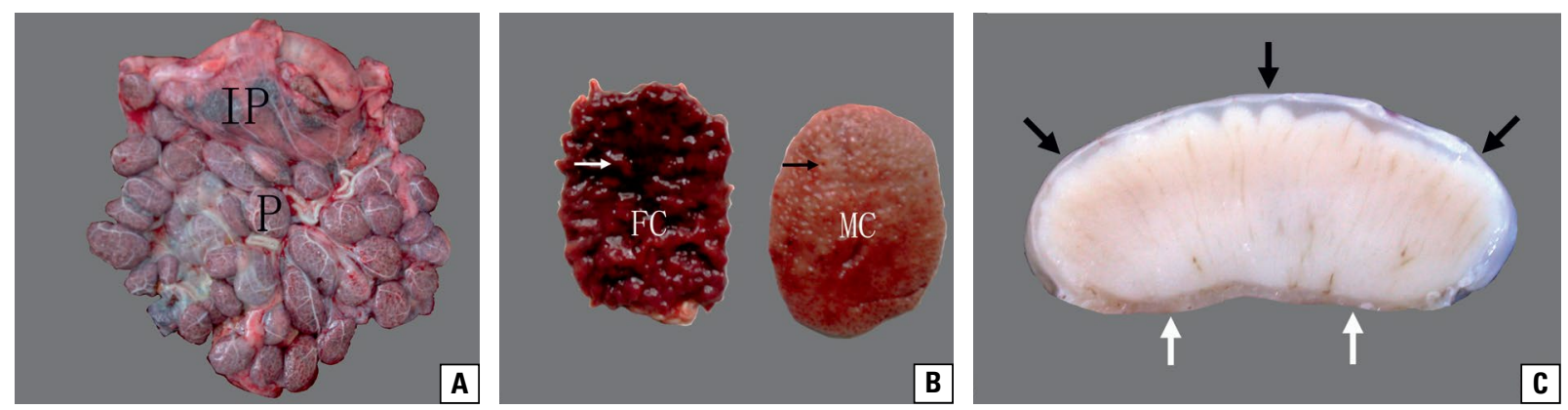

Figure 1. Anatomical observation of yak placenta during gestation; A. The yak placenta was composed of multiple separate placentomes (P) and interplacentomes (IP); B. The placentome was composed of foetal chorioallantois (FC) and maternal caruncles (MC); the cotyledonary villi (white arrow) were within the corresponding caruncular crypts (black arrow); C. The vertical section of the placentome; the dome-shaped placentome rested on a straight maternal plate (white arrows), and the chorionic plate (black arrows) covered the convex caruncle like a cap.

tal plate), which covered the maternal caruncle like a hat. The maternal tissue had a relatively straight substrate (maternal plate), which was located under the caruncle. Since the maternal plate of the placentome was relatively straight, no caruncle stalk was observed (Fig. 1C).

\section{Histology of the yak placentome}

Yak placentomes were composed of chorioallantois and uterine caruncles. The chorionic plate vertically projected the primary chorionic villi, and the latter divided into secondary branches. Then, divisions from secondary villi into tertiary villi could also be observed. The uterine caruncles were divided into many compartments (crypts) due to the invasion of chorionic villi. The invading chorionic villi inserted into the corresponding caruncular crypts, an interdigitation occurred (Fig. 2A, B). The inner layer of the chorionic villi was foetal mesenchyme, which had scattered mesenchymal cells and blood vessels. The outer layer was lined with a trophoblastic epithelium, which consisted of two different cell types: mononuclear trophoblast epithelial cells and trophoblast giant cells (TGCs). The mononuclear epithelial cells were irregular or cuboidal in shape and predominated in the trophoblastic epithelium, whose nuclei were oval or rather uneven in shape. TGCs were typically oval and appeared in different positions among mononuclear epithelial cells. One nucleus or two nuclei were usually visible in a single trophoblast giant cell. The nucleoli were distinct, and the cytoplasm was abundant and stained intensely (Fig. 2C).

The crypts of the yak placentome consisted of an inner layer of maternal connective tissues and the outer crypt epithelium. The crypt epithelium predominantly contained the uninucleate cryptal epithelial cells, and among the uninucleate cells, there were a few cryptal giant cells. The uninucleate epithelial cells were generally cuboidal in shape. The rounded or ovoid nuclei were situated in the centre of the cells. The nucleoli were rather indistinct, and the cytoplasm was stained lightly. Cryptal giant cells were larger than uninucleate epithelial cells, and their shapes were usually ovoid or irregular; they contained three or even more nuclei that almost filled the cells, so the cytoplasm was scarce. The maternal connective tissues were stained intensely and had abundant blood vessels (Fig. 2C). The straight maternal plate was located beneath the placentome, and there were plentiful uterine glands, blood vessels and a dense cellular layer in the endometrium lamina propria close to the maternal plate. The glandular epithelium was simple columnar or cuboidal, occasionally there existed secretory products in the glandular cavity.

\section{Histochemical observations of the yak placentome}

Paraffin sections of paraformaldehyde-fixed material were stained with PAS for neutral mucopolysaccharides and muco- and glycoprotein or were stained for acid mucopolysaccharide with Alcian blue ( $A B, p H ~ 2.5)$. The AB-PAS ( $p H ~ 2.5)$ reaction was used to separate acid glycoconjugates from neutral types. Foetal mesenchyma was essentially negative to the PAS reaction; positive $A B$ staining is indicated by a blue or light blue stain, and after the reaction with both dyes, the foetal mesenchyma appeared blue (Fig. 3A-C).

Trophoblast giant cells were strongly positive after the PAS reaction, and red granules were observed after incubation with the Schiff reagent. They were also stained light blue with $A B(\mathrm{pH} 2.5)$ and stained intensely reddish-purple or purple in the AB-PAS 

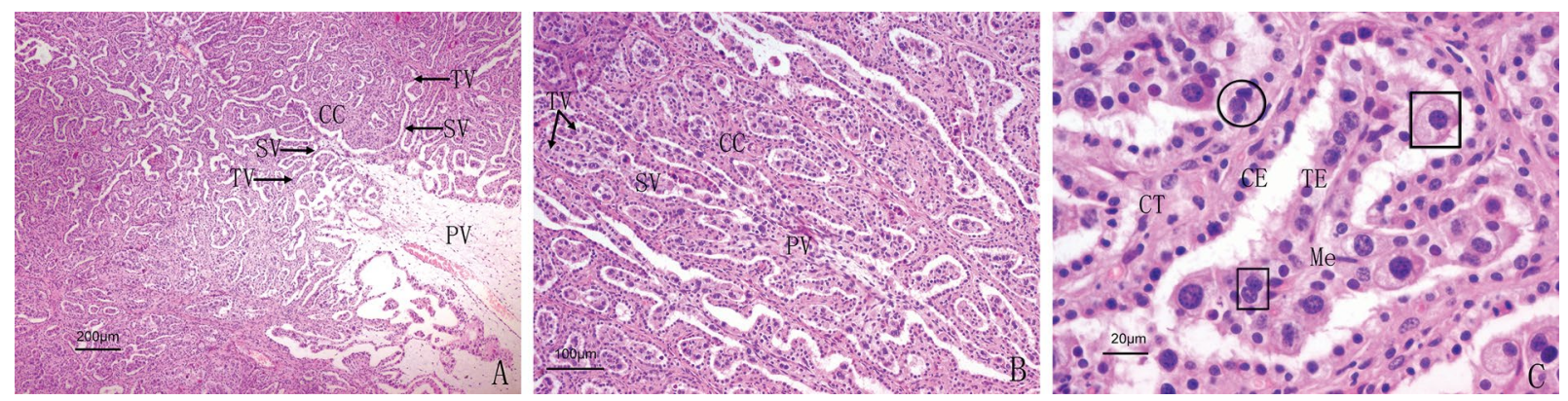

Figure 2. Histological section (H\&E) of yak placentome showing that the chorionic plate projected the primary villi (PV), secondary villi (SV) and tertiary villi (TV) into the corresponding caruncular crypt (CC). There were characteristic trophoblast giant cells (rectangles) and crypt giant cells (circle) in the trophoblast epithelium (TE) and crypt epithelium (CE); CT — connective tissue; Me — mesenchyme.
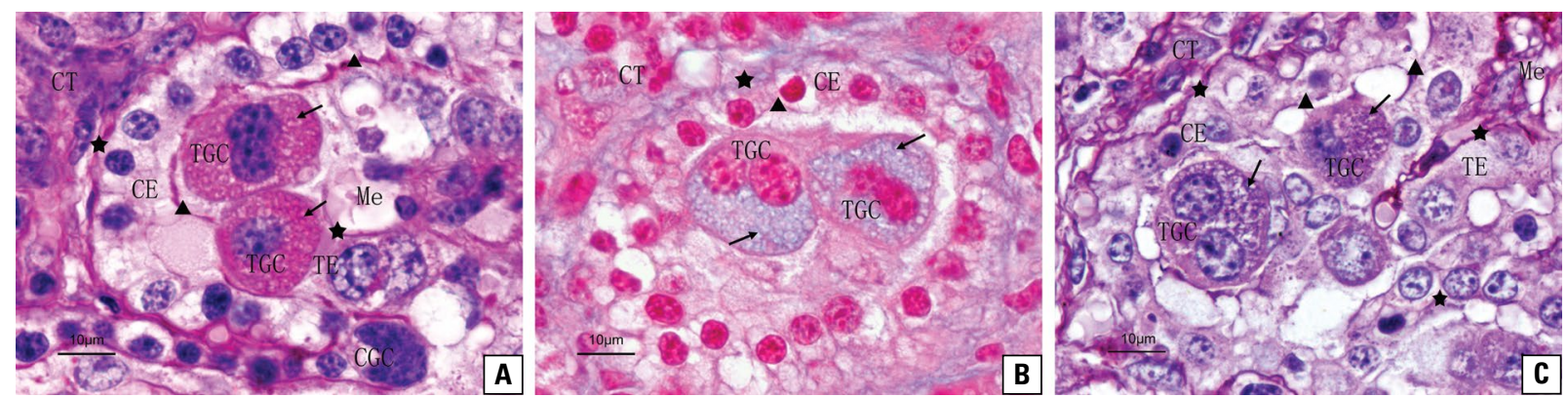

Figure 3. Distribution of glycoconjugate in yak placentome; A. The PAS reaction indicated that the red positive granules (arrowheads) filled the trophoblast giant cells (TGCs), and the positive reaction also appeared in the contact areas (triangles) between the crypt epithelium (CE) and trophoblast epithelium (TE) as well as in the basal lamina (asterisks) and maternal connective tissue (CT); Me — mesenchyme; CGC crypt giant cell; B. $A B(p H ~ 2.5)$ staining of the yak placentome had similar results to those of the PAS reaction; the positive areas appeared light blue; C. The intensely reddish-purple or purple positive areas were observed following the AB-PAS reaction.

$(\mathrm{pH} 2.5)$ reaction. The positive staining was either distributed in the entire cytoplasm of TGCs or restricted to one side of the cell (Fig. 3A-C). Mononuclear trophoblast epithelial cells and uninucleate cryptal epithelial cells remained unstained with PAS, $A B(\mathrm{pH} 2.5)$ or AB-PAS ( $\mathrm{pH}$ 2.5). Strongly positive staining (red) with the PAS reaction was observed in the junction regions between trophoblasts and the crypt epithelium, and light blue staining with $A B(\mathrm{pH} 2.5)$ or intensely reddish-purple or purple staining with $A B-P A S(p H$ 2.5) were also found. The staining properties of the basement membranes of both trophoblasts and the crypt epithelium were similar to that of the junction regions. Maternal connective tissues were stained red in the PAS reaction, light blue in the $A B(p H 2.5)$ reaction and light reddish-purple in the $A B$-PAS $(\mathrm{pH} 2.5)$ reaction (Fig. 3A-C).

\section{Transmission electron microscopy observations of the yak placentome}

Mononuclear trophoblast epithelial cells. Electron micrographs showed that the mononuclear trophoblast epithelial cells were usually columnar or irregular. The cytoplasmic membrane had a relatively smooth surface and was attached closely to the adjacent uninuclear trophoblast epithelial cells through tight junctions and desmosomes. The apical border had abundant microvilli interdigitating with microvilli of the corresponding uninucleate cryptal epithelial cells, forming the foetal-maternal contact zone. A great number of intracellular vesicles were present in the apical cytoplasm of the trophoblastic cells; sometimes, a few vesicles were also observed wrapped in the network of the microvilli. Their nuclei presented ovoid or irregular shapes and appeared either in a basal or central position. Abundant intranuclear euchromatins were distributed diffusely over the whole nuclear region, while a relatively few heterochromatins were organised in clumps mostly attached to the nuclear membrane. The cytoplasm contained regular organelles including mitochondria and endoplasmic reticulum. Most of the round or ovoid mitochondria were mainly concentrated in the apical and supranuclear cytoplasm, but a few were found beneath the 

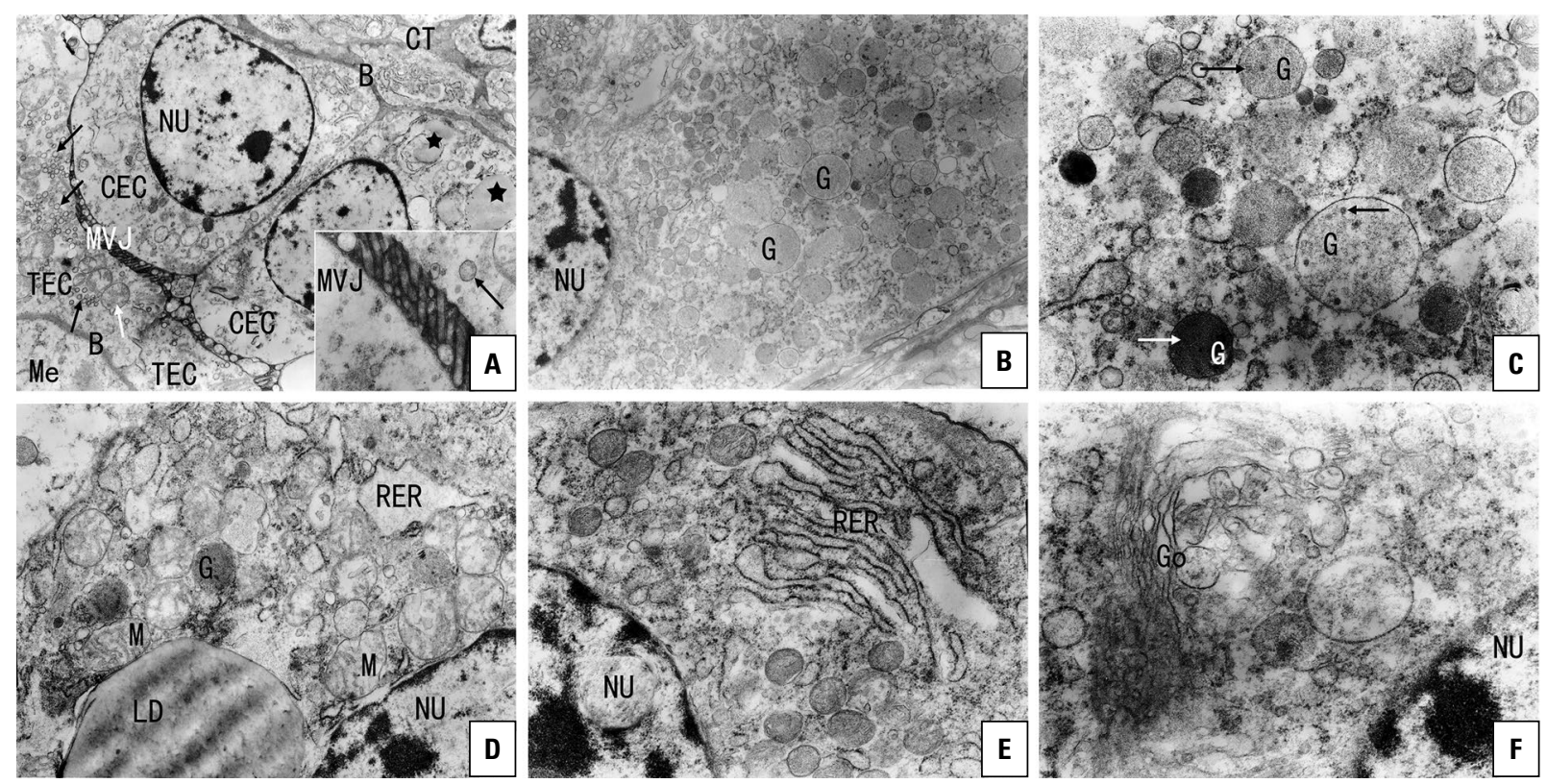

Figure 4. TEM micrographs of yak placentome; $\mathbf{A}$. The trophoblast and crypt epithelial cells (TEC and CEC) were connected via a microvillar junction (MVJ) and rested on a well-developed basement membrane (B). There were mitochondria and vesicles within the sub-microvillous cytoplasm of TECs. Some lipid droplets could be seen within the cytoplasm of the CEC; NU — nuclei; Me - mesenchyme; CT — connective tissue $(\times 4000)$; Inset: Showing MVJ and vesicles $(\times 15000)$; B. There were numerous cytoplasmic granules within the trophoblast giant cells $($ TGCS) $(\times 5000)$; C. Many granules of the TGCs contained microvesicles $(\times 15000)$; D. TGC, showing mitochondria (M) and lipid droplet (LD) $(\times 12000)$; E. TGC, showing rough endoplasmic reticulum (RER) $(\times 15000)$; F. TGC, showing Golgi complex $(\times 20000)$.

nucleus. The rough endoplasmic reticulum (RER) was dispersed throughout the cytoplasm. At the bottom of the cells, a well-developed basement membrane was distinctly seen, under which a foetal mesenchyme rich in capillaries was observed (Fig. 4A).

Trophoblast giant cells. The ovoid TGCs usually contained a smooth cell membrane and ovoid or round nuclei. The nuclei were generally located in the centre of the cells, sometimes situated in lateral cytoplasm. The heterochromatins of TGCs were organised in clumps mostly attached to the nuclear membrane, while a few were situated centrally in the nucleus. Furthermore, abundant euchromatins were distributed in the nucleoplasm (Fig. 4B). The cytoplasm of TGCs was stained intensely and contained abundant organelles, other than lipid droplets, that were characterised by the presence of many secretory granules, which were round or ovoid in shape, either distributed in the whole cytoplasm or concentrated toward one side of the cell. These secretory granules were completely surrounded by a membrane, grainy and of variable electron density. Some of them contained microvesicles of variable numbers (Fig. 4B-D). Cytoplasmic components also included abundant round or ovoid mitochondria. Many mitochondria were usually concentrated close to the endoplasmic reticulum and lipid droplets, and the others were distributed diffusely over the whole cytoplasm (Fig. 4D). In addition, the TGC cytoplasm also contained rough endoplasmic reticulum grouped in parallel arrays. Polyribosomes were observed on the surface of the RER or distributed throughout the cytoplasm, and free ribosomes were abundant through the cytoplasm (Fig. 4E). Additionally, the Golgi apparatus was well developed, arranged parallel to each other, and many vesicles and vacuoles of varying size were around each Golgi (Fig. 4F).

Uninucleate cryptal epithelial cells. The uninucleate cryptal epithelial cells were usually cuboidal in shape and had a smooth cytomembrane. Their nuclei appeared round or slightly irregular and were usually located in the basal zone of the cells, but some were situated in the middle of the cell. Many euchromatins were seen in the whole nucleoplasm, while heterochromatins were mostly attached to the nuclear membrane. The cytoplasm contained mitochondria, which were round or ovoid in shape and mainly distributed in the supranuclear cytoplasm. The cytoplasm also contained rough endoplasmic reticulum, free ribosomes, and polyribosomes. The 

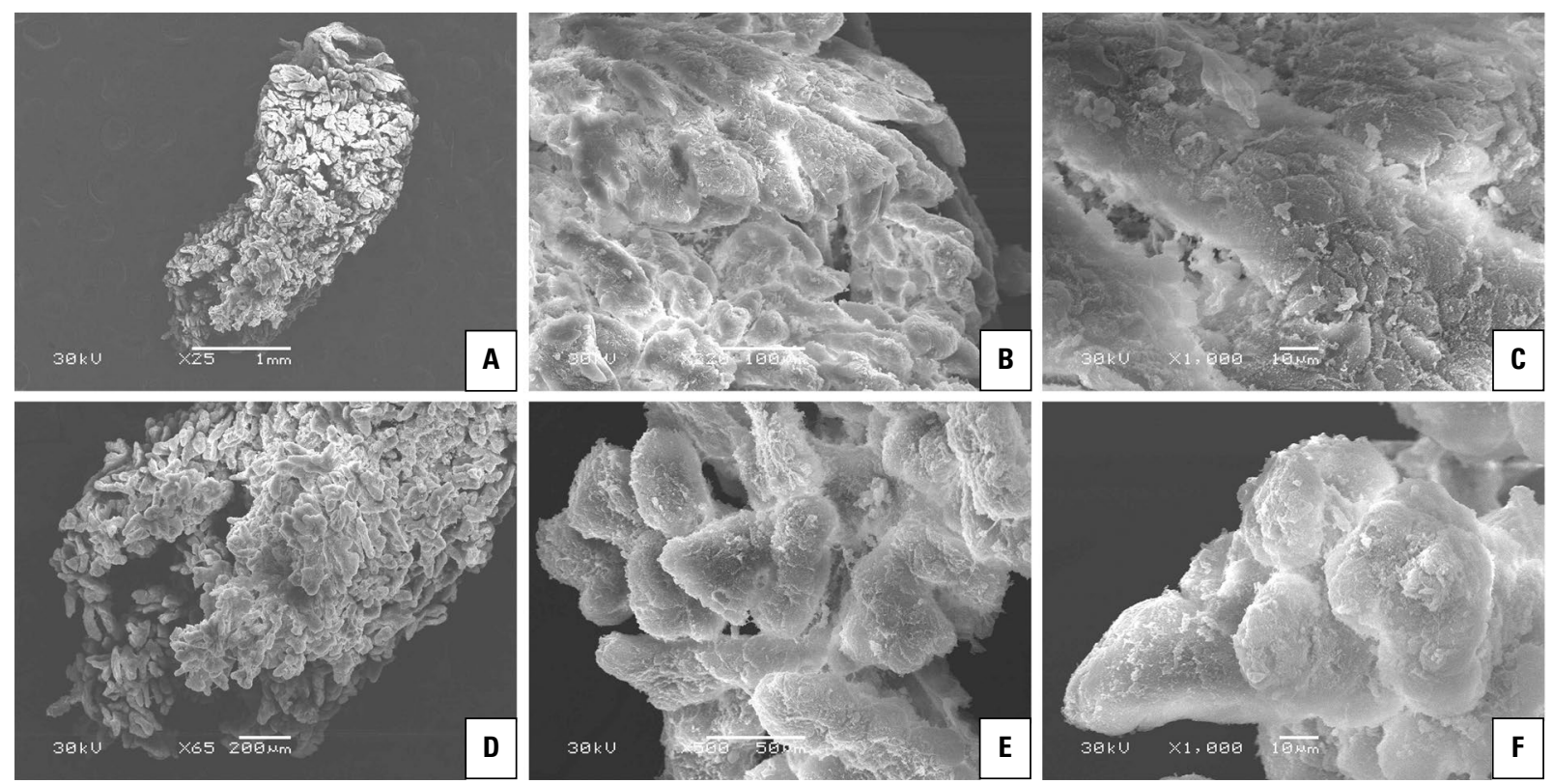

Figure 5. Scanning electron microscopy (SEM) micrographs of yak placentome; A. SEM micrograph of a single villous tree in yak; B. Enlargement of the central part of the villous trees shown in panel A. Secondary villi branches from the stem villi are arranged in layers; C. Note the long and slender tertiary villi that are covered with trophoblast cells; D. Enlargement of the tip of the villous trees shown in panel A. A single villous tree dividing into several stem villi; $\mathbf{E}$. Note that the simple (bulbous) appearance of secondary and tertiary villi; F. SEM micrograph of simple tertiary villi in yak.

Golgi apparatus was poorly developed and rarely observed. Several lipid droplets of different sizes were occasionally found in the supranuclear cytoplasm. The apical part of the uninucleate cryptal epithelial cells contained numerous microvilli, forming a contact zone with mononuclear trophoblast epithelial cells. However, there was no microvillar junction between the uninucleate cryptal epithelial cells and TGCs. These cells were based on well-developed basement membranes that were closed to the maternal connective tissues (Fig. 4A).

\section{Scanning electron microscopy observations of the yak placentome}

The SEM showed that a single villous tree of the yak placentome displayed conical or triangular shapes and could be described as a Christmas tree. A wider base of the Christmas tree close to the chorionic plate was observed but gradually became slender along its course. Additionally, at the villous tip, the stem villus frequently divided into two or more parallel, vertical terminal branches, which were generally thinner in diameter than the villus of origin (Fig. 5A). Yak chorionic villi branched to the third order. The stem villi, which were in the central part of the Christmas tree or close to the chorionic plate, projected relatively long and large secondary villi at approximately right angles, and most secondary villi were leaf-like and arranged in layers. Then, the secondary villi also projected numerous long and slender tertiary villi (Fig. 5B), which were covered with trophoblast cells (Fig. 5C). In contrast to the villus in the central part or close to the chorionic plate, the simple appearance of secondary and tertiary villi could be seen at the tip of the yak villous tree (Fig. 5D). The shorter and smaller secondary villi were generally projected at approximately right angles or slightly acute from each primary villus, and simple and bulbous tertiary villi covered with trophoblast cells was observed (Fig. 5E, F).

\section{DISCUSSION}

From this investigation, we concluded that the villous-crypt architecture pattern of placentomes was basically similar to different bovid genera. However, some obvious differences in the shape of a single villous tree and their ramification pattern were observed in each species. First, similar to cattle, yaks have a conical or Christmas tree-like shape villous tree [12, 13]. However, the villous trees of African buffalo and water buffalo placentomes were often described as a "Tuscany-cypress" shape due to their slender form and rudimentary branching $[1,19]$. Secondly, villous 
branching patterns in African buffalo were distinctly less complex than that in cattle and yak and more closely resembled features of the water buffalo. In African buffalo and water buffalo, there were two types of villous trees, a rough type and a smooth type $[1,19]$. However, the smooth type of villous tree was not found in the placentomes of yak and cattle $[12,19]$. Furthermore, the secondary and tertiary villi of African buffalo and water buffalo appeared simple and bulbous; in contrast, the secondary villi of yak and cattle were long and slender and equipped with distinct, pointed tertiary villi $[1,12,19]$. Stem villi projected villi of denser arrangements at approximately right angles or slightly acute, which resulted in the maximal functional surface area of villous tissue in a given space [12]. Thirdly, our present study found that at the villous tip, the stem villi frequently divided into two or more parallel, vertical terminal branches. Schmidt et al. [19] reported similar results in African buffalo and indicated that the division from the villous tip into several terminal branches occurred frequently in African buffalo but seldom in cattle. Additionally, Miglino et al. [15] carried out a study on cloned placentae in cattle and found that one villous tree projected more than one primary villus occupying each crypt, as opposed to usually one primary villus of the cotyledon in each primary crypt of the caruncle in non-cloned placentae. The total volume of terminal villi, the "working part" of villous trees, distinctly increased because the stem villi might contribute to enhancing the efficiency of transplacental diffusional exchange [12].

Two different cell types had been recognised in the trophectoderm of the placenta of ruminant animals: mononucleate trophoblast cells and trophoblast giant cells $[9,18]$. Similar results were found in the present study. Trophoblast giant cells of yak villus appeared typically oval and usually contained one or two nuclei; TGCs with more than two nuclei described by Klisch et al. [9] in cattle but absent in yak might suggest that serial sections should be further examined. In his fundamental work on TGC development, Wimsatt [23] describes binuclear TGCs that developed from mononuclear TGCs by acytokinetic mitoses in which mitotic figures were much larger than those in mitoses of normal mononuclear trophoblast cells. This is an early hint that TGC nuclei may be polyploid because the size of the mitotic figures depends on the number or size of the chromosomes they contain. The functional significance of polyploidization is supposed to be an increased synthetic capacity of TGCs, which results from the elevated number of gene copies available for transcription. In multinuclear cells, the transport between nucleus and cytoplasm could be facilitated by a higher ratio of nuclear surface to nuclear volume [9]. For example, the mouse giant cell trophoblast layer is adjacent to the decidua zone, where the trophoblast giant cells are parietal trophoblast giant cells (P-TGCs), which are the largest mononuclear polyploid cells, and the number of ploidy can reach $1024 \mathrm{n}$ (normal tissue cell are biploid, which is $2 n,[20])$. Their volume is approximately 16 times that of sponge trophoblast cells and more than 100 times that of glycogen cells [11], and they can secrete vascular endothelial growth factor and proliferin to promote vascular hyperplasia and adrenomudullin [28], NOS [5] and anticoagulant factor [22] to expand blood vessels and promote maternal blood flow to the placenta.

The ultrastructure features of TGCs of yak placentomes were similar to those in cow, sheep, goats, deer, African buffalo and Tragulidae [19, 24, 25], including abundant mitochondria, rough endoplasmic reticulum, well-developed Golgi bodies and many characteristic membrane-bound granules. According to the excellent research published in recent years [24, $26,27]$, these granules contain numerous protein and glycoprotein constituents, such as placental lactogen, prolactin-related protein I and pregnancy-associated glycoproteins. The contents of the TGC granules are delivered into the maternal compartments following migration and fusion of TGCs with maternal endometrial epithelial cells [24]. In addition, TGCs are thought to produce steroid hormones such as oestradiol and progesterone $[8,14,21]$. In yaks, TGCs were characterizsed by the presence of many lipid droplets, serving as a store of low-density lipoprotein cholesterol, which was the principle substrate for the biosynthesis of steroid hormones [16].

Noticeably, the granules of TGCs in yaks were positive to periodic acid Schiff (PAS, appeared red) staining and Alcian blue ( $A B$, appeared blue) staining, showing that they contained neutral and acidic glycoconjugates; similar chromogenic reactions were discovered in the special granules of other ruminants $[15,24,27]$. In yaks, the positive reactions also presented in the microvillar junction regions between trophoblasts and the crypt epithelium and in the maternal connective tissues. Interestingly, the distribution patterns of positive reactions to the two dyes 
in yaks were very consistent with the distribution of pregnancy associated glycoproteins (PAGs) in cows studied by Wooding et al. [26]. According to their research, the more modern PAGs were expressed primarily in trophoblast binucleate cells and seen in the stromal layer within the maternal caruncles, whereas the more ancient PAGs were localised largely in the contact regions of the trophectoderm and uterine epithelium. The PAGs expressed in yaks should be further studied using relevant techniques in the future.

Electron microscopy examination in our present study on yak placentomes showed that mononucleate trophoblast cells had morphological characteristics relating to specialised functions primarily involved in substance exchange, including the presence of numerous mitochondria, abundant microvilli and large numbers of vesicles in the apical cytoplasm of the cells. The microvilli interdigitated with the corresponding microvilli arising from the crypt epithelial cells, forming the foeto-maternal contact zone $[2,6]$, which could provide an increased surface area for the absorption of materials. Plentiful vesicles in the apical cytoplasm of the cells might indicate pinocytotic activity delivering exchange material, and the mitochondria appeared to be related to substance exchange $[2,6]$.

\section{CONCLUSIONS}

In conclusion, the morphological structures of yak placentomes were similar to those of other bovid genera; however, certain differences were observed. These findings might provide morphological evidence for evolutionary relationships between different bovid genera. The presence of a relatively complex villous-crypt architecture pattern, special absorptive cells and characteristic endocrine TGCs in yak placentomes are indispensable for foetal survival and development during gestation.

\section{Acknowledgements}

We thank Junwei Hu and Hua Zhang for their assistance with collecting samples.

\section{Funding}

Grant sponsor: the National Natural Science Foundation of China (No. 31472244), the Natural Science Foundation of Gansu Province (No. 17JR5RA141), the Fuxi Foundation of Youth Talent at Gansu Agricultural University (No. Gaufx-02Y10). Scientific research foundation for new Scholars, Gansu Agricul- tural university (No. GSAU-RCZX201701), Innovation foundation of the College of Veterinary Medicine, Gansu Agriculture University (No. JYCX-KX016), and National key research and development programme (No. 2017YFD0502200).

\section{REFERENCES}

1. Abd-Elnaeim MMM, Miglino MA, Pfarrer C, et al. Microvascular architecture of the fetal cotyledons in water buffaloes (Bubalus bubalis) during different stages of pregnancy. Ann Anat. 2003; 185(4): 325-334, doi: 10.1016/ S0940-9602(03)80053-5, indexed in Pubmed: 12924470.

2. Björkman $\mathrm{NH}$. Light and electron microscopic studies on cellular alterations in the normal bovine placentome. Anat Rec. 1969; 163(1): 17-29, doi: 10.1002/ar.1091630103, indexed in Pubmed: 5763133.

3. Cui Y, Yu SJ. An anatomical study of the internal genital organs of the yak at different ages. Vet J. 1999; 157(2): 192-196, doi: 10.1053/tvjl.1998.0283, indexed in Pubmed: 10204417.

4. Farin PW, Crosier AE, Farin CE. Influence of in vitro systems on embryo survival and fetal development in cattle. Theriogenology. 2001; 55(1): 151-170, doi: 10.1016/ s0093-691x(00)00452-0, indexed in Pubmed: 11198080.

5. Gagioti S, Scavone C, Bevilacqua E. Participation of the mouse implanting trophoblast in nitric oxide production during pregnancy. Biol Reprod. 2000; 62(2): 260-268, doi: 10.1095/biolreprod62.2.260, indexed in Pubmed: 10642561.

6. Guillomot M, Guay P. Ultrastructural features of the cell surfaces of uterine and trophoblastic epithelia during embryo attachment in the cow. Anat Rec. 1982; 204(4): 315-322, doi: 10.1002/ar.1092040404, indexed in Pubmed: 7181136.

7. Hafez ESE, Hafez B. Reproduction in farm animals. Lippincott Williams and Wilkins., Philadelphia 2000.

8. Hoffmann B, Schuler G. The bovine placenta; a source and target of steroid hormones: observations during the second half of gestation. Domest Anim Endocrinol. 2002; 23(1-2): 309-320, indexed in Pubmed: 12142247.

9. Klisch K, Pfarrer C, Schuler G, et al. Tripolar acytokinetic mitosis and formation of feto-maternal syncytia in the bovine placentome: different modes of the generation of multinuclear cells. Anat Embryol (Berl). 1999; 200(2): 229-237, indexed in Pubmed: 10424879.

10. Knechtel C. Brunstverhalten beim Kaffernbuffel (Syncerus caffer caffer) im Tierpark Berlin Friedrichsfelde. Zool Gart. 1993; 63: 32-58.

11. Lee SJ, Talamantes F, Wilder E, et al. Trophoblastic giant cells of the mouse placenta as the site of proliferin synthesis. Endocrinology. 1988; 122(5): 1761-1768, doi: 10.1210/ endo-122-5-1761, indexed in Pubmed: 3359962.

12. Leiser R, Krebs C, Klisch K, et al. Fetal villosity and microvasculature of the bovine placentome in the second half of gestation. J Anat. 1997; 191 (Pt 4): 517-527, doi: 10.1046/j.14697580.1997.19140517.x, indexed in Pubmed: 9449071.

13. Leiser R, Pfarrer $C$, Abd-Elnaeim $M$, et al. Feto-maternal anchorage in epitheliochorial and endotheliochorial placental types studied by histology and microvascular corrosion casts. Trophoblast Research. 1998; 12: 21-39. 
14. Matamoros RA, Caamano L, Lamb SV, et al. Estrogen production by bovine binucleate and mononucleate trophoblastic cells in vitro. Biol Reprod. 1994; 51(3): 486-492, doi: 10.1095/biolreprod51.3.486, indexed in Pubmed: 7803620.

15. Miglino MA, Pereira FTV, Visintin JA, et al. Placentation in cloned cattle: structure and microvascular architecture. Theriogenology. 2007; 68(4): 604-617, doi: 10.1016/j.theriogenology.2007.04.060, indexed in Pubmed: 17568663.

16. Njogu A, Owiti GO, Persson E, et al. Ultrastructure of the chorioallantoic placenta and chorionic vesicles of the lesser bush baby (Galago senegalensis). Placenta. 2006; 27(6-7): 771-779, doi: 10.1016/j.placenta.2005.07.003, indexed in Pubmed: 16174532.

17. Qian F. Livestock embryology. China Science and Culture Press, Hong Kong 2003.

18. Schlafer DH, Fisher PJ, Davies CJ. The bovine placenta before and after birth: placental development and function in health and disease. Anim Reprod Sci. 2000; 60(4): 145-160, indexed in Pubmed: 10844191.

19. Schmidt S, Gerber D, Soley JT, et al. Histo-morphology of the uterus and early placenta of the African buffalo (Syncerus caffer) and comparative placentome morphology of the African buffalo and cattle (Bos taurus). Placenta. 2006; 27(8): 899-911, doi: 10.1016/j.placenta.2005.09.008, indexed in Pubmed: 16293303.

20. Simmons DG, Fortier AL, Cross JC. Diverse subtypes and developmental origins of trophoblast giant cells in the mouse placenta. Dev Biol. 2007; 304(2): 567-578, doi: 10.1016/j. ydbio.2007.01.009, indexed in Pubmed: 17289015.

21. Wango EO, Heap RB, Wooding FB. Progesterone and 5 beta-pregnanediol production by isolated fetal placental binucleate cells from sheep and goats. J Endocrinol. 1991; 129(2): 283-289, doi: 10.1677/joe.0.1290283, indexed in Pubmed: 2040862.
22. Weiler-Guettler H, AirdW C, Rayburn H. Developmentally regulated gene expression of thrombomodulin in postimplantanion mouse embryos. Development. 1996; 122(7): 2271-2281.

23. Wimsatt WA. Observations on the morphogenesis, cytochemistry, and significance of the binocleate giant cells of the placenta of ruminants. Am J Anat. 1951; 89(2): 233-281, doi: 10.1002/aja.1000890204, indexed in Pubmed: 14894441.

24. Wooding FB. Current topic: the synepitheliochorial placenta of ruminants: binucleate cell fusions and hormone production. Placenta. 1992; 13(2): 101-113, indexed in Pubmed: 1631024.

25. Wooding FBP, Kimura J, Fukuta K, et al. A light and electron microscopical study of the Tragulid (mouse deer) placenta. Placenta. 2007; 28(10): 1039-1048, doi: 10.1016/ /j.placenta.2007.04.010, indexed in Pubmed: 17597203.

26. Wooding FBP, Roberts RM, Green JA. Light and electron microscope immunocytochemical studies of the distribution of pregnancy associated glycoproteins (PAGs) throughout pregnancy in the cow: possible functional implications. Placenta. 2005; 26(10): 807-827, doi: 10.1016/j. placenta.2004.10.014, indexed in Pubmed: 16226131.

27. Yamada O, Todoroki J, Kizaki K, et al. Expression of prolactin-related protein $I$ at the fetomaternal interface during the implantation period in cows. Reproduction. 2002; 124(3): 427-437, indexed in Pubmed: 12201816.

28. Yotsumoto $S$, Shimada $T$, Cui $C Y$, et al. Expression of adrenomedullin, a hypotensive peptide, in the trophoblast giant cells at the embryo implantation site in mouse. Dev Biol. 1998; 203(2): 264-275, doi: 10.1006/ dbio.1998.9073, indexed in Pubmed: 9808778.

29. Yu SJ, Huang YM, Chen BX. Reproductive patterns of the yak. I. Reproductive phenomena of the female yak. Br Vet J. 1993; 149(6): 579-583, doi: 10.1016/S00071935(05)80042-9, indexed in Pubmed: 8111618. 